\title{
Much ado about the family veto
}

\author{
Ian M. Ball, MD, MSc $\cdot$ Simon Oczkowski, MD, MSc $\cdot$ Robert Sibbald, MSc $\mathbb{D}$
}

Received: 28 October 2020/Revised: 3 December 2020/Accepted: 3 December 2020/Published online: 10 February 2021

(C) Canadian Anesthesiologists' Society 2021

Many people believe that, once a person has properly registered themselves as an organ donor, their wishes to donate will always be respected. Nevertheless, once in a while, surrogate decision-makers (SDMs) will override (or veto) the donor's stated wishes and prevent organ donation from occurring. As clinicians and a clinical ethicist with an academic and clinical interest in organ donation and transplantation, we were intrigued by the article written by Anthony et al., in this issue of the Journal, entitled "Family veto in organ donation: the experiences of organ and tissue donation coordinators in Ontario". ${ }^{1}$

This research team conducted semi-structured interviews with Ontario Organ and Tissue Donation Coordinators (OTDCs) to better understand their experiences and perspectives regarding family vetoes for organ donation. Although only ten OTDCs participated in the study, the research team ensured the OTDCs were experienced at their role, and that there was a diversity of age and practice location.

This qualitative research follows a recent survey and editorial published in the Journal on the same subject., ${ }^{2,3}$ $\mathrm{We}$ are pleased to see increased discussion of this

\footnotetext{
I. M. Ball, MD, MSc

Department of Medicine and Department of Epidemiology and Biostatistics, Western University, London, ON, Canada

S. Oczkowski, MD, MSc

Department of Medicine, McMaster University, Hamilton, ON, Canada

R. Sibbald, MSc ( $\square)$

Clinical Ethics, London Health Sciences Centre, London, ON,

Canada

e-mail: robert.sibbald@lhsc.on.ca
}

important topic in the literature. It raises many important themes.

It is not explicitly stated by the authors, but for a family veto to occur, the patient's wish to be an organ donor must have been known, by some form of communication. This previous knowledge may be from discussions between the patient and their SDM or by the patient's registered wish to be an organ donor.

While the highlighted issues are important: increasing the donor pool, patient advocacy, emotional distress, and supporting grieving families, we would like to emphasize the importance of patient autonomy. As the authors correctly state: "SDMs have no legal authority to withhold organ donation authorization if the deceased donor had previously consented". ${ }^{1}$

It is commendable that well-meaning healthcare team members go to great lengths to comfort families at their time of grief. Indeed, some OTDCs in the study recognized the occasional "acceptable veto." Nevertheless, the clinical team members' duty, even after death, is first and foremost to the patient. It is not logical that our commitments to a patient should change when neurologic death is determined, but this likely occurs because of a motivation to care for families and/or out of fear of the repercussions of defying family wishes. The patient remains in the same bed and their body continues to be cared for to ensure their organs can be maintained. This is unlike other matters of estate, which are sometimes changed by courts after death so as to not reflect the patient's prior wishes. It was therefore disheartening to see that, in the Anthony et al. study, some healthcare providers were reported to feel ambivalent about organ donation. In an ideal world, their patient advocacy (and acceptance of pre-specified donation wishes) would continue unabated after neurologic death determination, and clinicians would 
feel supported in following through with the patient's wish to donate.

In addition to a legal requirement to uphold the wishes of the patient, we would argue that the healthcare team members are ethically bound to respect the patient's wish to donate organs, if possible. For this reason, we are concerned about any discussion that preserves and normalizes the family veto, as a practice contrary to patient autonomy. The only ethically acceptable family vetoes are those where a family has a compelling reason to think that the patient would have changed their mind about the decision to donate. While family distress could influence a patient's donation decision, family distress alone is insufficient to override patients' autonomous wishes. Instead, we should better support families in understanding the donation process, particularly as many families who refuse organ donation regret their decision months later. 4

We can take solace knowing that, in most cases, healthcare team members are able to both uphold patient autonomy and comfort grieving families. At the same time, the numerous challenges described in the study suggest that changes to the donor consent process are needed to better balance the autonomous decisions of patients with the needs of families. For instance, explicitly informing registered organ donors that their wish will only be adhered to if family also agrees with the donation decision is an option. While this approach would provide transparency on how donor registration is enacted in practice, and uphold the principles of informed consent, it falls short of true patient autonomy, as potential donors may disagree with decisions made by their family at the bedside. Better information for registered donors about what donation entails would also strengthen the argument that registered donation truly represents informed consent and should be adhered to unless there is good reason to think the donor would have deviated from the choice do donate.

Discussions with SDMs about organ donation should be framed to make it clear that clinicians are not offering a choice, but instead informing SDMs of patients' wishes. In circumstances where families wish to veto known patient wishes to become organ donors, firm but compassionate discussion is usually enough to convince families to respect their loved one's wishes, even at the cost of short-term personal emotional hardship. In cases when families do not respond positively to an intensivist's guidance, a difficult conversation becomes necessary. This creates an extremely challenging situation for families and intensive care unit (ICU) staff, and could put clinicians at risk of regulatory college complaints (or even litigation), which can have a profound impact even if the clinician is ultimately judged to have been correct. The avoidance of uncomfortable conversations with families results in suboptimal shared decision-making in the ICU. In the case of organ donation, the harm is magnified, affecting not only patient care but also potential recipients who are prevented from receiving a life-saving gift.

To address our concerns, we feel it important to engage a broader discussion about how family veto came to be respected in the first place. It seems that there are three distinct possibilities. The first is that OTDCs never crafted a policy in the first place, reasonably hoping that good conversations would resolve most conflicts and that the harms associated with family veto would be minimal (thus undeserving of formal policy). If this were the case, we hope that attention to the harms associated with violating patient autonomy and losing viable organs might influence OTDCs to adopt a more intentional approach.

The second possibility is that OTDCs crafted a policy that respects family veto because they feel the moral obligation to the family outweighs any commitment made to a now-deceased patient. We feel we have sufficiently undermined this rationale above.

The last possibility is that OTDCs crafted a policy that respects family veto because of the potential for negative media attention that could be drawn to the donation movement should a family make their disagreement public. Based on this assumption, it would be reasonable to apply a utilitarian analysis in preserving the larger organ donation agenda - i.e., could more lives be saved by avoiding a potential drop in donor registration following bad press? The assumption that media attention would negatively impact the societal organ donation rate is speculative and requires greater scrutiny. It is conceivable that a betterinformed public, and a policy that always respects patient choice, would in fact lead to increased donation rates. The problem with this rationale for respecting family veto is that it takes the path of least resistance, avoiding a broader difficult conversation with the public at large, while at the same time not giving a charitable interpretation as to how they would respond. Ultimately, even if the worst-case scenario resulted from respecting patient choice, and donation rates were negatively impacted, we would rather accept this outcome following good process.

As participants of the deceased donation symposium at the 2017 Canadian Critical Care Forum (CCCF), we reflect on a speaker who asked the audience of several hundred intensivists how many ask SDMs for organ donation provide consent in cases where the patient's wish to donate is known. Unfortunately, during the informal survey of intensivists at the $\mathrm{CCCF}$, many responded that they request a decision from SDMs, when the decision is not the SDMs' to make. Reflecting the OTDC's prioritization of language clarity, it is important that intensivists move away from asking SDMs for consent in these cases; but instead inform 
them what the next steps in the organ donation practice will be, in keeping with the patient's previously expressed wishes and preserving their autonomy. Adopting an "informed assent" approach acknowledges the patient's wishes and the impact of donation upon the family, and it allows for the possibility of the rare "acceptable vetoes" where a potential donor may have changed their mind.

Participants of the Anthony et al. study described moral distress in cases where family vetoes superseded the patient's wishes. This is not surprising vis-à-vis our fiduciary responsibility to the patient. Improved collaboration between the care team and the OTDCs, as well as increased support for OTDCs and ICU staff when they must advocate for SDMs to uphold the donor's previously expressed wishes will help to reduce moral distress, increase the number of times that patient autonomy is respected, and increase the number of organs available to recipients. The goal of increased organ donation articulated at the end of the Anthony et al. study is certainly noble and one we wholeheartedly support; but it should come second to the shared duty of protecting patient autonomy.

Beaucoup de gens croient qu'une fois qu'un individu s'est correctement inscrit comme donneur d'organes, ses volontés seront toujours respectées. Néanmoins, il arrive parfois que les personnes autorisées à décider au nom d'autrui passeront outre (ou mettront leur veto) aux souhaits déclarés du donneur et empêcheront le don d'organes de se produire. En tant que cliniciens et éthicien clinique ayant un intérêt académique et clinique pour le don et la transplantation d'organes, nous avons été intrigués par l'article d'Anthony et coll., publié dans ce numéro du Journal, intitulé « Veto familial au don d'organes : expériences des coordonnateurs en don d'organes et de tissus en Ontario ». ${ }^{1}$

Cette équipe de recherche a mené des entrevues semistructurées de coordonnateurs en don d'organes et de tissus (CDOT) ontariens afin de mieux comprendre leurs expériences et leurs points de vue concernant les vetos familiaux au don d'organes. Bien que seulement dix CDOT aient participé à l'étude, l'équipe de recherche s'est assurée que les CDOT avaient de l'expérience dans leur fonction et que ce groupe avait une diversité d'âge et de lieu de pratique.

Cette recherche qualitative fait suite à une enquête et un éditorial récents publiés dans le Journal sur le même sujet. $^{2,3}$ Nous sommes heureux de voir que ce sujet important est davantage abordé dans la littérature. En effet, il soulève de nombreuses problématiques importantes.

Bien que cela ne soit pas énoncé de façon explicite par les auteurs, pour qu'il y ait un veto familial, il faut que le désir du patient d'être donneur d'organes ait été connu, par une certaine voie de communication. Cette connaissance $\mathrm{du}$ souhait antérieur du patient peut avoir fait l'objet de discussions entre le patient et la personne autorisée à décider en son nom, ou être la volonté documentée et enregistrée du patient d'être un donneur d'organes.

Bien que les enjeux mis en évidence soient importants augmentation du bassin de donneurs, défense des droits des patients, détresse émotionnelle et soutien aux familles en deuil -, nous tenons à souligner l'importance de l'autonomie des patients. Comme l'affirment à juste titre les auteurs : « Les personnes autorisées à décider au nom d'autrui n'ont pas le pouvoir légal de refuser l'autorisation du don d'organes si le donneur décédé y avait déjà consenti ».

Il faut féliciter les membres bien intentionnés de l'équipe de soins de santé qui mettent beaucoup d'énergie à réconforter les familles endeuillées. En effet, certains CDOT dans l'étude ont reconnu qu'occasionnellement ils faisaient face à un «veto acceptable ». Néanmoins, l'obligation des membres de l'équipe clinique, même après la mort, est d'abord et avant tout envers le patient. Il n'est pas logique que nos engagements envers un patient changent lorsqu'un diagnostic de décès neurologique est posé; toutefois, de tels cas existent probablement en raison d'un engagement à prendre soin des familles et/ou par crainte des répercussions possibles lorsqu'on défie les volontés de la famille. Le patient reste dans le même lit et son corps continue d'être soigné pour s'assurer que ses organes puissent être maintenus. Cela n'a rien à voir avec d'autres questions de succession, qui sont parfois modifiées par les tribunaux après le décès de telle façon qu'elles ne reflètent pas les souhaits antérieurs d'un patient. Il était donc décourageant de voir que, dans l'étude d'Anthony et coll., certains fournisseurs de soins de santé ont rapporté être ambivalents envers le don d'organes. Dans un monde idéal, leurs plaidoyers en faveur des patients (et l'acceptation de leur souhait préétabli en matière de don) se poursuivraient sans relâche après un diagnostic de décès neurologique, et les cliniciens se sentiraient soutenus dans l'exécution des souhaits du patient en matière de don.

En plus d'une l'obligation juridique de respecter les souhaits du patient, nous soutenons que les membres de l'équipe de soins de santé sont éthiquement tenus de respecter le désir du patient de faire don de ses organes, si possible. Pour cette raison, toute discussion qui préserve et normalise le veto familial en tant que pratique contraire à l'autonomie des patients nous inquiète. Les seuls vetos familiaux acceptables d'un point de vue éthique sont ceux où une famille invoque une raison convaincante de penser que le patient aurait changé d'avis au sujet de sa décision de faire un don. Bien que la détresse familiale puisse influencer la décision de don d'un patient, elle ne suffit pas 
à elle seule pour annuler le souhait autonome des patients. Au lieu de cela, nous devrions soutenir les familles afin qu'elles comprennent mieux le processus du don, d'autant plus que de nombreuses familles qui refusent le don d'organes regrettent leur décision des mois plus tard. ${ }^{4}$

Nous pouvons nous consoler en sachant que, dans la plupart des cas, les membres de l'équipe de soins de santé sont à la fois en mesure de maintenir l'autonomie des patients et de réconforter les familles endeuillées. En même temps, les nombreux défis décrits dans l'étude suggèrent que des changements au processus de consentement du donneur sont nécessaires afin de mieux équilibrer les décisions autonomes des patients et les besoins des familles. Par exemple, une option consisterait à informer explicitement les donneurs d'organes enregistrés que leur souhait ne sera respecté que si la famille est également d'accord avec leur décision de don. Bien que cette approche garantirait la transparence de la façon dont l'enregistrement du consentement des donneurs est appliqué dans la pratique et respecterait les principes du consentement éclairé, elle ne reflèterait toutefois pas l'autonomie réelle des patients, car les donneurs potentiels peuvent être en désaccord avec les décisions prises par leur famille à leur chevet. De meilleures informations pour les donneurs enregistrés sur ce que le don implique renforceraient également l'argument selon lequel le don enregistré représente véritablement un consentement éclairé qui devrait être respecté, à moins qu'il n'y ait de bonnes raisons de penser que le donneur n'ait modifié son choix.

Les discussions avec les personnes autorisées à décider au nom d'autrui au sujet du don d'organes devraient être encadrées afin d'établir clairement que les cliniciens n'offrent pas le choix, mais plutôt les informent des souhaits des patients. Dans les cas où les familles souhaitent opposer leur veto aux volontés connues des patients de devenir donneurs d'organes, une discussion à la fois ferme et empreinte de compassion suffit généralement à convaincre les familles de respecter les volontés de leur proche, même au prix d'une détresse émotionnelle personnelle de courte durée. Dans les cas où les familles ne répondent pas positivement aux conseils d'un intensiviste, une conversation difficile devient alors nécessaire. Cela crée une situation extrêmement difficile tant pour les familles que pour le personnel des unités de soins intensifs (USI) et pourrait mettre les cliniciens à risque de plaintes aux organismes de réglementation (ou même de litiges). Une telle situation peut avoir un impact profond, même s'il est finalement jugé que le clinicien avait raison. Si l'on évite les conversations délicates avec les familles, cela diminue la qualité d'une prise de décision partagée aux soins intensifs. Dans le cas du don d'organes, le préjudice est amplifié, affectant non seulement les soins aux patients, mais aussi les receveurs potentiels, empêchés de recevoir un don salvateur.

Pour tenir compte de nos inquiétudes, nous pensons qu'il est important d'engager une discussion plus large sur la façon dont le veto familial est devenu accepté en premier lieu. Il semble qu'il y ait trois possibilités distinctes. La première est que les CDOT n'ont jamais élaboré de politique, espérant raisonnablement qu'une bonne conversation résoudrait la plupart des conflits et que les préjudices associés au veto familial seraient minimes (ce qui rendrait donc inutile une politique formelle). Si tel est le cas, nous espérons que l'attention portée aux préjudices associés à la violation de l'autonomie du patient et à la perte d'organes viables pourrait influencer les CDOT à adopter une approche plus délibérée.

La deuxième possibilité est que les CDOT ont élaboré une politique qui respecte le veto familial parce qu'ils estiment que l'obligation morale envers la famille est plus importante que tout engagement pris envers un patient aujourd'hui décédé. Nous estimons avoir suffisamment discrédité une telle justification ci-dessus.

La dernière possibilité est que les CDOT ont élaboré une politique qui respecte le veto familial en raison du risque de faire l'objet d'une attention négative des médias, laquelle pourrait se concentrer sur le principe des dons si une famille décidait de rendre public son désaccord. Basé sur cette hypothèse, il serait raisonnable d'appliquer une analyse utilitaire pour préserver le programme plus large de don d'organes - c'est-à-dire, se pourrait-il qu'un nombre plus élevé de vies soient sauvées en évitant une baisse potentielle de l'enregistrement des donneurs à la suite d'une mauvaise presse? La présomption selon laquelle l'attention des médias aurait un impact négatif sur le taux de don d'organes dans la société n'est que spéculation et nécessite un examen plus approfondi. Il est tout à fait concevable qu'un public mieux informé et une politique qui respecte toujours le choix des patients entraînent en fait en une augmentation du nombre de dons. Le problème avec cette justification du respect du veto familial est qu'il s'agit de la voie de moindre résistance, qui esquive une conversation difficile élargie avec le grand public, tout en ne lui accordant pas une interprétation philanthropique quant à la façon dont il réagirait. En fin de compte, même si le pire des scénarios résultait du respect du choix des patients et que les taux de dons étaient affectés négativement, nous préférerions accepter ce résultat suite à un processus optimal.

En tant que participants au Symposium sur les dons après décès tenu lors du Forum canadien des soins intensifs (CCCF) 2017, nous pensons à un conférencier qui a demandé à un auditoire de plusieurs centaines d'intensivistes combien d'entre eux demandaient leur consentement aux personnes autorisées à décider au nom 
d'autrui en matière de don d'organes dans les cas où la volonté du patient de faire un don était connue. Malheureusement, au cours de ce sondage informel auprès d'intensivistes lors du CCCF, beaucoup ont répondu qu'ils demandaient une décision des personnes autorisées à décider au nom d'autrui, alors que la décision n'est pas de leur ressort. Étant donné l'importance capitale accordée à la précision linguistique par les CDOT, il est crucial que les intensivistes cessent de demander leur consentement aux personnes autorisées à décider au nom d'autrui dans de tels cas; plutôt, il conviendrait de les informer des prochaines étapes du processus de don d'organes, conformément aux volontés précédemment exprimées par le patient et en préservant ainsi leur autonomie. L'adoption d'une approche d' « approbation éclairée » reconnaît à la fois les souhaits du patient et l'impact du don sur la famille, et cela laisse la place à un possible mais rare « veto acceptable » lorsqu'un donneur potentiel pourrait avoir changé d'avis.

Les participants de l'étude d'Anthony et coll. ont décrit leur détresse morale dans les cas où les vetos familiaux ont annulé et remplacé les volontés du patient. Ce n'est pas surprenant compte tenu de notre responsabilité de mandataire envers le patient. C'est en améliorant la collaboration entre l'équipe de soins et les CDOT, ainsi qu'en offrant un soutien accru aux CDOT et au personnel de l'USI lorsqu'ils doivent plaider auprès des personnes autorisées à décider au nom d'autrui afin qu'elles respectent les souhaits précédemment exprimés par le donneur, que nous parviendrons à réduire la détresse morale, à augmenter les situations dans lesquelles l'autonomie des patients est respectée, et à augmenter le nombre d'organes à la disposition des receveurs. L'objectif d'une augmentation des dons d'organes articulé à la fin de l'étude d'Anthony et coll. est certainement noble et nous le soutenons sans réserve; mais il ne devrait pas être plus important que la responsabilité partagée de protéger l'autonomie des patients.

Disclosures None.

Funding statement None.

Editorial responsibility This submission was handled by Dr. Philip M. Jones, Deputy Editor-in-Chief, Canadian Journal of Anesthesia.

Déclaration Aucune.

Déclaration de financement Aucune.

Responsabilité éditoriale Cet article a été traité par Dr Philip M. Jones, rédacteur en chef adjoint, Journal canadien d'anesthésie.

\section{References}

1. Anthony SJ, Lin J, Pol SJ, Wright L, Dhanani S. Family veto in organ donation: the experiences of organ and tissue donation coordinators in Ontario. Can J Anesth 2021; DOI: https://doi.org/ 10.1007/s12630-021-01928-0.

2. Weiss MJ, English SW, D'Aragon F, et al. Survey of Canadian intensivists on physician non-referral and family override of deceased organ donation. Can J Anesth 2020; 67: 313-23.

3. Ball IM, Sibbald R, Oczkowski S. Family override for organ donation. Can J Anesth 2020; 67: 286-8.

4. Burroughs TE, Hong BA, Kappel DF, Freedman BK. The stability of family decisions to consent or refuse organ donation: would you do it again? Psychosom Med 1998; 60: 156-62.

Publisher's Note Springer Nature remains neutral with regard to jurisdictional claims in published maps and institutional affiliations. 